\title{
Laryngeal Mechanisms During Human 4-kHz Vocalization Studied With CT, Videostroboscopy, and Color Doppler Imaging
}

\author{
*Chen-Gia Tsai, †Yio-Wha Shau, $\ddagger$ Hon-Man Liu, and *Tzu-Yu Hsiao \\ Taipei, Taiwan
}

\begin{abstract}
Summary: The objective of this study was to investigate the underlying laryngeal mechanisms during the specific human $4-\mathrm{kHz}$ vocalization. The laryngeal configuration during this vocalization was measured using high-resolution computerized tomographic scan and videostrobolaryngoscopy. The color Doppler imaging (CDI) of medical ultrasound was used to detect the vibrations of glottal and supraglottal mucosa. During the $4-\mathrm{kHz}$ vocalization, the ventricular folds were adducted in the shape of a bimodal chink and the vocal folds were shaped as a "V" with an opening at the posterior glottis. In the coronal view, the laryngeal ventricles had collapsed and a divergent shaped conduit was observed at the posterior portion of the larynx. The surface mucosa vibration detected by CDI was noted over the bilateral ventricular folds and aryepiglottic folds. The vibration displacement was estimated to be on the order of $0.1 \mathrm{~mm}$. This vibration amplitude was too small to be detected in videostrobolaryngoscopy. The laryngeal configuration and CDI data suggested a diffuser jet with periodic vorticity bursts in the larynx producing $4 \mathrm{kHz}$ voice.
\end{abstract}

Key Words: Larynx-Color Doppler imaging-4-kHz vocalizationDiffuser-Vortex-induced surface vibration.

Accepted for publication October 10, 2006.

This study was approved by the Institution Review Board (No. 9561703001), National Taiwan University Hospital, Taipei, Taiwan.

This research was supported by Grant NSC 93-2811-B-002-079 and NSC 93-2314-B-002-113, Taiwan.

From the *Department of Otolaryngology, National Taiwan University, College of Medicine, National Taiwan University, Taipei, Taiwan; †Institute of Applied Mechanics, National Taiwan University, Taipei, Taiwan; and the $\doteqdot$ Department of Radiology, National Taiwan University Hospital, College of Medicine, National Taiwan University, Taipei, Taiwan.

Address correspondence and reprint requests to $\mathrm{Tzu}-\mathrm{Yu}$ Hsiao, MD, PhD, Department of Otolaryngology, National Taiwan University Hospital, College of Medicine, National Taiwan University \#7, Chung-San South Road, Taipei 100, Taiwan. E-mail: tzuyu@ha.mc.ntu.edu.tw

Journal of Voice, Vol. 22, No. 3, pp. 275-282

0892-1997/\$34.00

(C) 2008 The Voice Foundation doi:10.1016/j.jvoice.2006.10.007

\section{INTRODUCTION}

Very high-frequency vocalizations have attracted a great deal of attention from bioacousticians. Such vocalizations play a key role in acoustic communication of several species of birds ${ }^{1}$ and cetacean mammals. ${ }^{2}$ In the field of phoniatrics, the very high-frequency voice was discovered in research on canine vocalizations and identified as "whine" or "whistle-like sound." 3-5 Some human-specific singing techniques also rely on laryngeal vibrations with fundamental frequency $\left(F_{0}\right)$ exceeding $1 \mathrm{kHz}$. One example is the voice of the "flageolet" register, with frequency normally ranging from 1 to $1.5 \mathrm{kHz} .{ }^{4,6,7}$ In extreme cases, the frequency can even extend to $4 \mathrm{kHz} .^{8}$ Another example probably relevant to very high-frequency vocalization is 
"overtone singing" or "throat singing." An overtone singer can produce both a low drone at regular $F_{0}$ voice and a prominent high-pitched overtone at frequency $n F_{0}$ ( $n=6-13$ in typical performances) at the same time. ${ }^{9}$ In frequency spectral analysis, the high pitch tone arises from a very sharp formant centered at the $n$th overtone with frequency at 1$2.5 \mathrm{kHz}$. A "double-source" theory of overtone singing asserts that the significant overtone with the frequency of $n F_{0}$ is produced by a second sound source. The narrowing of the ventricular folds can produce a whistle-like voice, for example. ${ }^{10}$

The aim of this article was to study the underlying laryngeal mechanisms in a human $4-\mathrm{kHz}$ vocalization. To study this vocalization, measurement techniques capable of detecting high-frequency, small-amplitude tissue vibrations without interfering with the normal phonation process were needed. The color Doppler imaging (CDI) of medical ultrasound is an ideal tool to investigate such vocalization mechanisms. It detects tissue velocity rather than displacement under normal phonation conditions. In our previous studies, the CDI has been used to measure the frequency and the length of the vocal folds during normal phonation. ${ }^{11,12}$ In this study, a computerized tomographic (CT) scan and videostrobolaryngoscopy were used firstly to depict the glottal configuration, and then the CDI was applied to detect the tissue vibration velocity and areas in the larynx. A diffuser jet with vortex-induced surface vibration was proposed to explain the phonation mechanisms.

\section{MATERIALS AND METHODS}

One of the authors (CGT), a healthy man aged 34 years, was able to voluntarily prolong a sound from the larynx of approximately $4 \mathrm{kHz}$. The tongue was in its neutral position as for schwa. The sound was recorded with a microphone (AT9440, AudioTechnica, Tokyo, Japan) for spectral analysis. The intensity was measured with a precision sound level meter (B\&K 2235; Bruel\&Kjaer, Naerum, Denmark) placed at about $15 \mathrm{~cm}$ from the lips.

\section{High-resolution CT scan}

This study was performed with a commercially available, high-resolution CT scanner (Lightspeed16,
Advantage Workstation, version 4.2, General Electric, Milwaukee, WI) with a tube current of $250 \mathrm{~mA}$ and voltage of $120 \mathrm{kV}$. The scans were taken under conditions of quiet respiration, normal phonation, and $4 \mathrm{kHz}$ phonation. The scan region was from the hyoid level to the upper trachea. Image reconstruction was performed using a standard kernel. The region of the larynx and hypopharynx was reconstructed with a large field view of $18 \mathrm{~cm}$ and a slice thickness of $0.625 \mathrm{~mm}$.

\section{Videostrobolaryngoscopy}

The videostrobolaryngoscopic instruments included a stroboscope with halogen light source, a CCD camera, and a $70^{\circ}$ laryngeal telescope (Model 9200; KayPENTAX, Lincoln Park, NJ). The examination was performed without topical anesthesia. The subject tolerated the whole procedure well. The digital images were recorded during modal, falsetto, and $4-\mathrm{kHz}$ vocalizations.

\section{Color Doppler imaging}

A regular medical high-resolution US scanner (HDI-5000; ATL, Bothell, WA) with two lineararray transducers (L12-5 $38 \mathrm{~mm}$ and CL10-5 $25 \mathrm{~mm}$; ATL) was used. In the color mode, the pulse-repetition rate was $10,000 \mathrm{~Hz}$ and the measuring velocity range was set at $0-102.6 \mathrm{~cm} / \mathrm{s}$ with baseline offset. The frame rate in this condition was $7 \mathrm{~Hz}$. The US scan head was placed in coronal and horizontal planes, respectively, at the midline of the thyroid cartilage lamina on the right side.

\section{RESULTS}

The spectra of a $4-\mathrm{kHz}$ vocalization showed a prominent spectral component at $3.5-4.5 \mathrm{kHz}$ and broadband noise filtered by the vocal tract (Figure 1). The intensity of the second and the third harmonics was always weaker than that of the $F_{0}$ with reductions of more than $40 \mathrm{~dB}$. Its sound pressure level was $60 \pm 2 \mathrm{~dB}$ measured at about $15 \mathrm{~cm}$ from the lips.

During the $4-\mathrm{kHz}$ vocalization, the ventricular folds were adducted in the shape of a bimodal chink (anterior and posterior openings). The glottis was shaped like a "V," with a tight closure in the anterior portion and an opening in the posterior glottis 
A
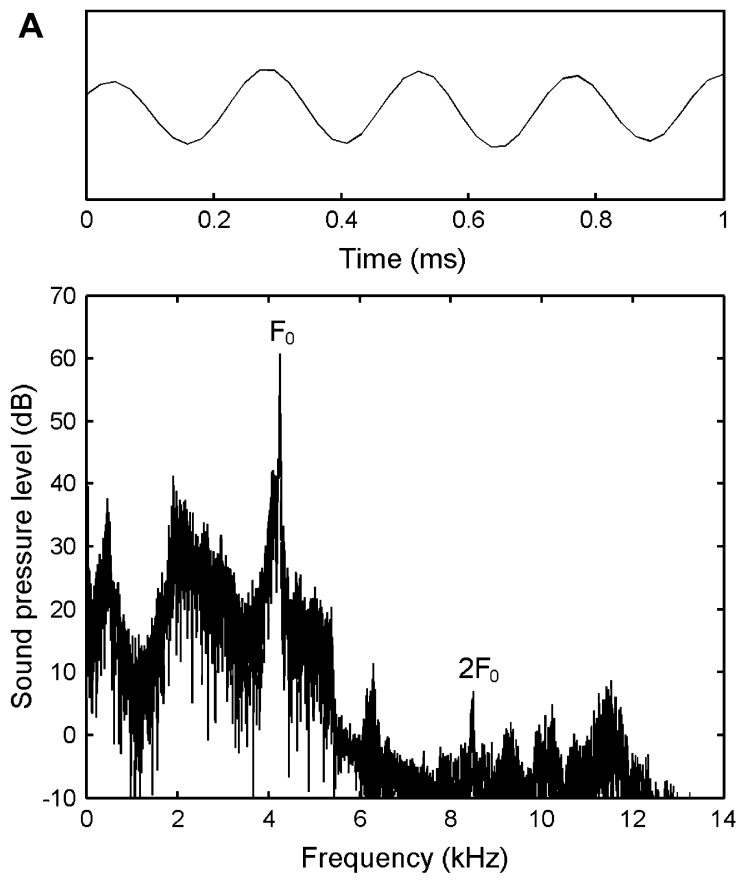

B
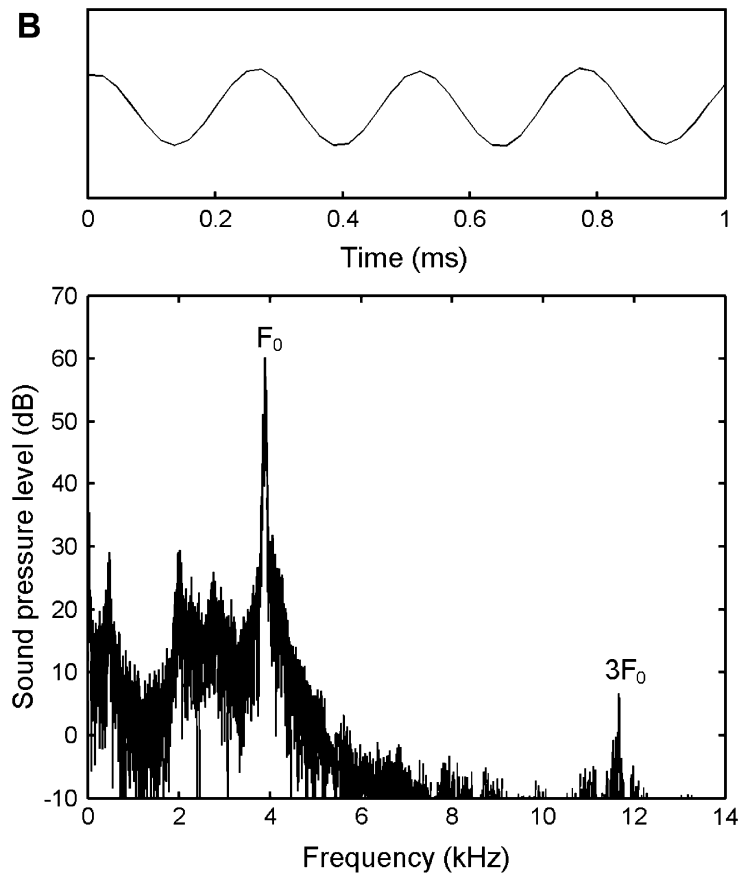

FIGURE 1. Sound waveforms and frequency spectra of human $4 \mathrm{kHz}$ vocalization. Fast Fourier transformation size $=16,384$ points. A. $F_{0}=3889 \mathrm{~Hz}$. B. $F_{0}=4239 \mathrm{~Hz}$. The second and the third harmonics were always weaker than the $F_{0}$ with reductions of more than $40 \mathrm{~dB}$.

(Figure 2A). In the sagittal view, the adducted arytenoids in the outlet of the posterior glottis led the conduit running superior-anteriorly (Figure 2B). In the coronal view, the bilateral ventricles had collapsed. The ventricular folds together with the posterior glottis formed a divergent orifice with the angle of approximately $6^{\circ}$ during the $4-\mathrm{kHz}$ vocalization (Figure 2C). This divergent configuration was approximately located at the posterior onethird of the glottis. Reconstructed 3D images showed a complicated geometry of the air channel (Figure 2D).

In videostrobolaryngoscopy, the mucosal wave of the vocal folds can be easily demonstrated in modal and falsetto phonation. During the $4-\mathrm{kHz}$ vocalization, no mucosal wave was visible. In the imaging, the bimodal-chink configuration of the ventricular folds was also confirmed (Figure 3). During the examination, the vocal tract above the larynx was fully opened.

In the coronal CDI, the color artifact along the vibrating surface can be found on the air-mucosa interface of the previously described channel confined by the posterior glottis, ventricular folds, and arytenoids during the $4-\mathrm{kHz}$ vocalization (Figure 4B). The total vertical length of the color artifacts was approximately $1 \mathrm{~cm}$.

The color code in CDI allowed for the estimation of the vibration displacement on the mucosal surface during the $4-\mathrm{kHz}$ vocalization. It was assumed that the displacement of the mucosal surface vibration was sinusoidal:

$$
y(t)=y_{0} \cos \left(2 \pi F_{0} t\right) .
$$

The color code in CDI represented the average speed $\left(v_{0}\right)$ of the top-half of $y(t)$ (the rectified sine wave). ${ }^{11,12}$ It is given by

$$
v_{0}=2 \pi F_{0} y_{0} \frac{\int_{0}^{\pi} \sin \theta \mathrm{d} \theta}{\pi}=4 F_{0} y_{0} .
$$

According to the color code in CDI, $v_{0}$ was approximately the upper limit of CDI $(102.6 \mathrm{~cm} / \mathrm{s})$. The mucosal surface vibration displacement $2 y_{0}$ can be estimated as $0.125 \mathrm{~mm}$ for $F_{0}=4 \mathrm{kHz}$. This vibration amplitude was too small to be detected in videostrobolaryngoscopy. 

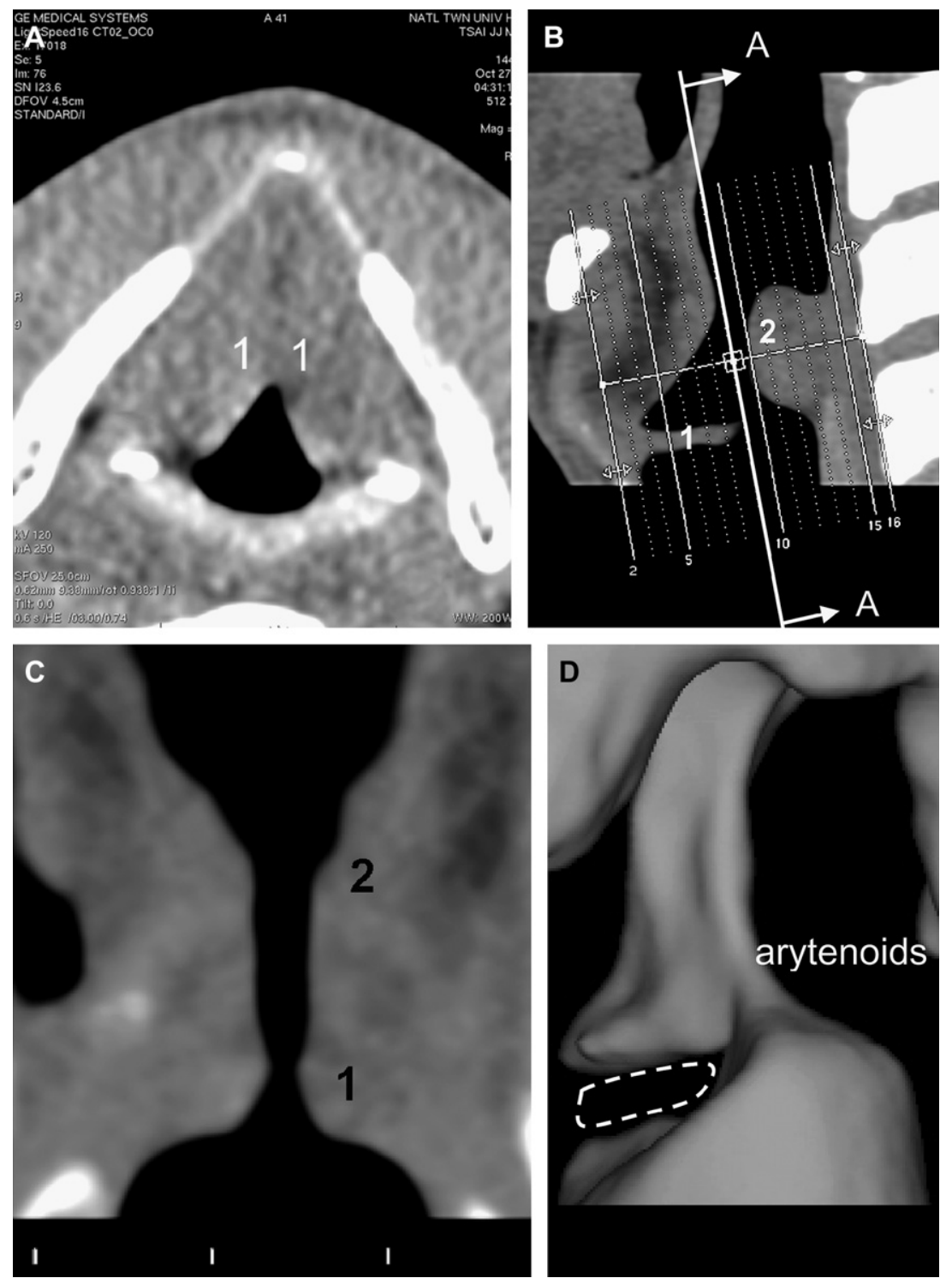

FIGURE 2. CT imaging during the $4-\mathrm{kHz}$ vocalization. A. Horizontal section view of CT imaging at vocal fold level. While the membranous portion of the vocal folds was closed, there was a posterior opening between the arytenoids. B. Sagittal section view. C. AA coronal section view. The laryngeal ventricles collapsed and a divergent configuration was observed at the posterior-third of the larynx. D. Reconstructed 3D image of the air channel in the larynx, as seen from left-behind. (1: vocal folds' level; 2: superior part of arytenoids; dash line: the tight closure in the anterior portion of the vocal folds).

In horizontal views, the air-mucosa interface of the ventricular fold can be identified as a curved white line. On this white line, the vibration color artifacts appeared constant during the $4-\mathrm{kHz}$ vocalization (Figure 4D).

\section{DISCUSSION}

\section{Laryngeal configuration during $4-\mathrm{kHz}$ vocalization}

In this study, we examined the laryngeal configuration and small-amplitude mucosal vibrations 


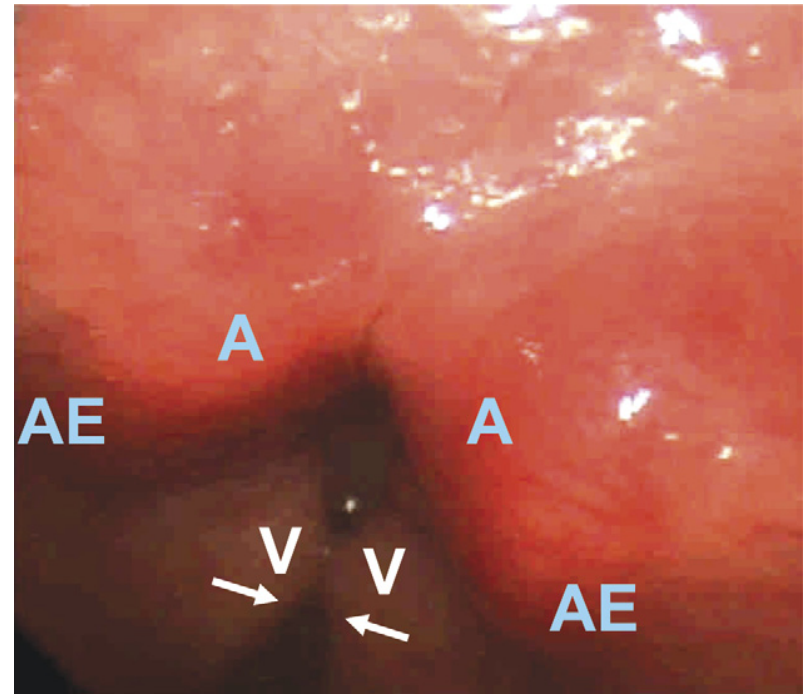

FIGURE 3. Videostrobolaryngoscopic image during the 4-kHz vocalization. V: ventricular folds; A: arytenoids; AE: aryepiglottic folds near arytenoids. The ventricular folds were adducted in the shape of a bimodal chink (anterior and posterior openings) and the bilateral arytenoids were shifted anteriorly.

during the 4-kHz vocalization. Data of CT imaging and videostrobolaryngoscopy pointed out that the ventricular folds were adducted in the shape of a bimodal chink and the glottis was shaped like a "V." It seemed that the air jet passed through the posterior glottis, ran superior-anteriorly and entered a very thin channel confined by the ventricular folds bilaterally and the arytenoids posteriorly (Figure 2B). It is interesting to note that the laryngeal configuration during the $4-\mathrm{kHz}$ vocalization shared similarity with whisper production. The "V" configuration of the vocal folds is one of the configurations during whispering, and medial supraglottal constriction has been found to play a key role in high-effort whispering. ${ }^{13}$ Indeed, during the preparation phase of the $4-\mathrm{kHz}$ vocalization the sound was characterized by whisper-like broadband noises. The laryngeal configuration during the 4$\mathrm{kHz}$ vocalization also resembled the configuration during Valsalva maneuver. ${ }^{14}$ This suggested that a small caliber of the conduit accompanied this production of $4 \mathrm{kHz}$ tones.

\section{Tone produced by diffuser flow}

At the posterior portion of the larynx, the reconstructed coronal images of CT showed a divergent orifice with an angle of approximate $6^{\circ}$ and length of about $1.5 \mathrm{~cm}$. This laryngeal geometry may be critical to the $4-\mathrm{kHz}$ vocalization. Similar highfrequency tones were generated by a steady airflow passing through a divergent orifice in an experimental study by Zhang et al. ${ }^{15}$ In their study, the jet tone was measured in three glottal orifice shapes: straight, divergent, and convergent profiles. In the straight and convergent orifices, the characteristics of the generated sound consisted of turbulence noises. In the divergent orifice (diffuser), high-frequency jet tones were produced at a low airflow rate. For a transglottal pressure of $6 \mathrm{~cm}$ $\mathrm{H}_{2} \mathrm{O}$, the frequency of the sound generated was $5.1 \mathrm{kHz}$. This jet tone was attributed to a hydrodynamic loop or diffuser-generated flow unsteadiness. The flow separation within the orifice was very sensitive to small disturbances, which may arise from the interactions between the vortices and the diffuser walls.

Although Zhang et al ${ }^{15}$ demonstrated that a constant airflow passing through a steady divergent glottal configuration can generate high-frequency tones, our study appears to have brought their research to practice. We found that the ventricular folds and vocal folds stacked together to obliterate the ventricles and form a divergent orifice at the posterior portion of the larynx. Videostrobolaryngoscopic and reconstructed CT imaging revealed that the laryngeal configuration was static, allowing the airflow to pass through as a diffuser flow. Although the anterior portion of the glottis was fully adducted during the $4-\mathrm{kHz}$ vocalization, they did not vibrate as in normal phonation. This phenomenon may be related to the collapse of the laryngeal ventricles. A similar laryngeal configuration with the laryngeal ventricles collapsed was also found during whispering. ${ }^{16}$

\section{Vortex-induced surface vibration of supraglottal mucosa}

Passing through the conduit formed by the posterior glottis, ventricular folds, and arytenoids, the air jet evoked small-amplitude, high-frequency surface vibrations of the supraglottal mucosa. In the coronal view, the color artifacts caused by the mucosal vibration often showed a two-sectional structure (Figure 4B). It is still impossible to verify the 
A

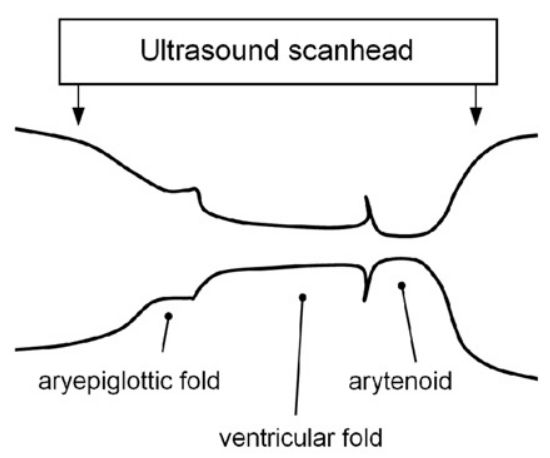

C

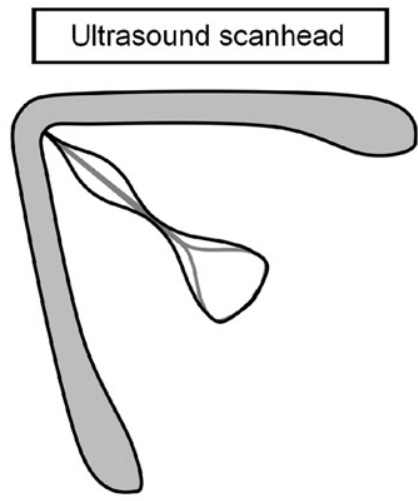

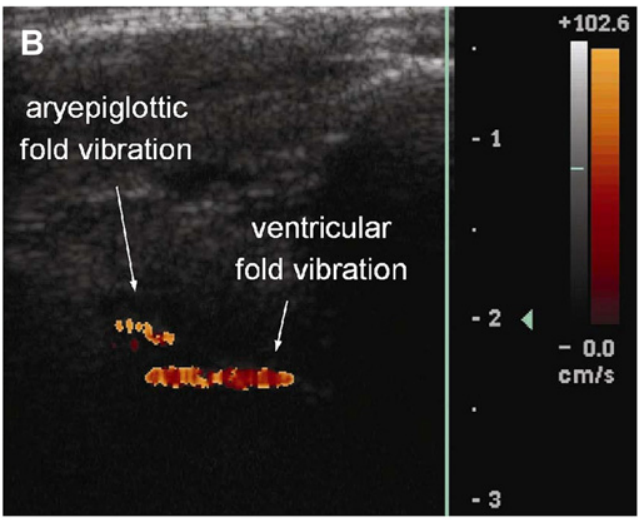

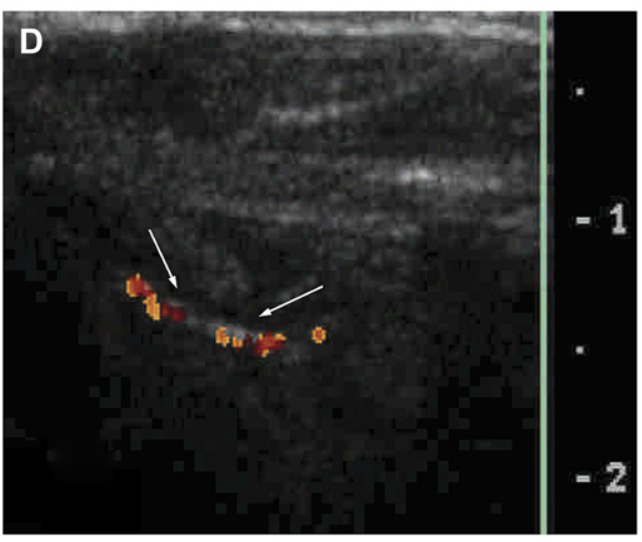

FIGURE 4. A. Schema of US coronal scan of the larynx. B. Coronal CDI view of US during the 4$\mathrm{kHz}$ vocalization. C. Schema of US horizontal scan of the larynx. D. Horizontal CDI view of US during the $4-\mathrm{kHz}$ vocalization at ventricular fold level. (Arrows: the curved white line) In coronal CDI, the color artifacts were found on the air-mucosa interface of the channel located in the posterior glottis. The two sections of the color artifacts in (B) may represent the surface vibrations of the ventricular fold and aryepiglottic fold, respectively.

precise anatomical sites of the two color code sections. It appears, however, that the long section of the color artifacts in Figure 4B represents the surface vibrations of the posterior glottis, and the short section represented the surface vibrations of the aryepiglottic folds near the arytenoids, or the mucosa covering the arytenoids structure.

During the $4-\mathrm{kHz}$ vocalization, the color artifacts caused by the mucosal vibrations always appeared in an orderly stripe pattern. According to our previous CDI research, ${ }^{11}$ this surface vibration was nearly periodic. It was possible that the frequency of mucosa surface vibration was the same as that of the produced tone. Hofmans et $\mathrm{al}^{17}$ investigated the unsteady flow through in vitro models of the glottis. Numerical results revealed that vortex distribution in the diffuser region showed a complex interaction of vortices and the diffuser walls. Bursts of vorticity leave the walls at regular intervals in time. The periodic surface vibration of the supraglottal mucosa during the $4-\mathrm{kHz}$ vocalization may be related to the vortex shedding induced by a diffuser jet. It may be that the high-frequency vibration of supraglottal mucosa observed in our experiments was due to the pressure fluctuations generated by periodic vorticity bursts.

The small-amplitude vibrations of supraglottal mucosa were likely to affect the sound production because flow separation is very sensitive to disturbances. In the study by Zhang et al, ${ }^{15}$ the divergent 

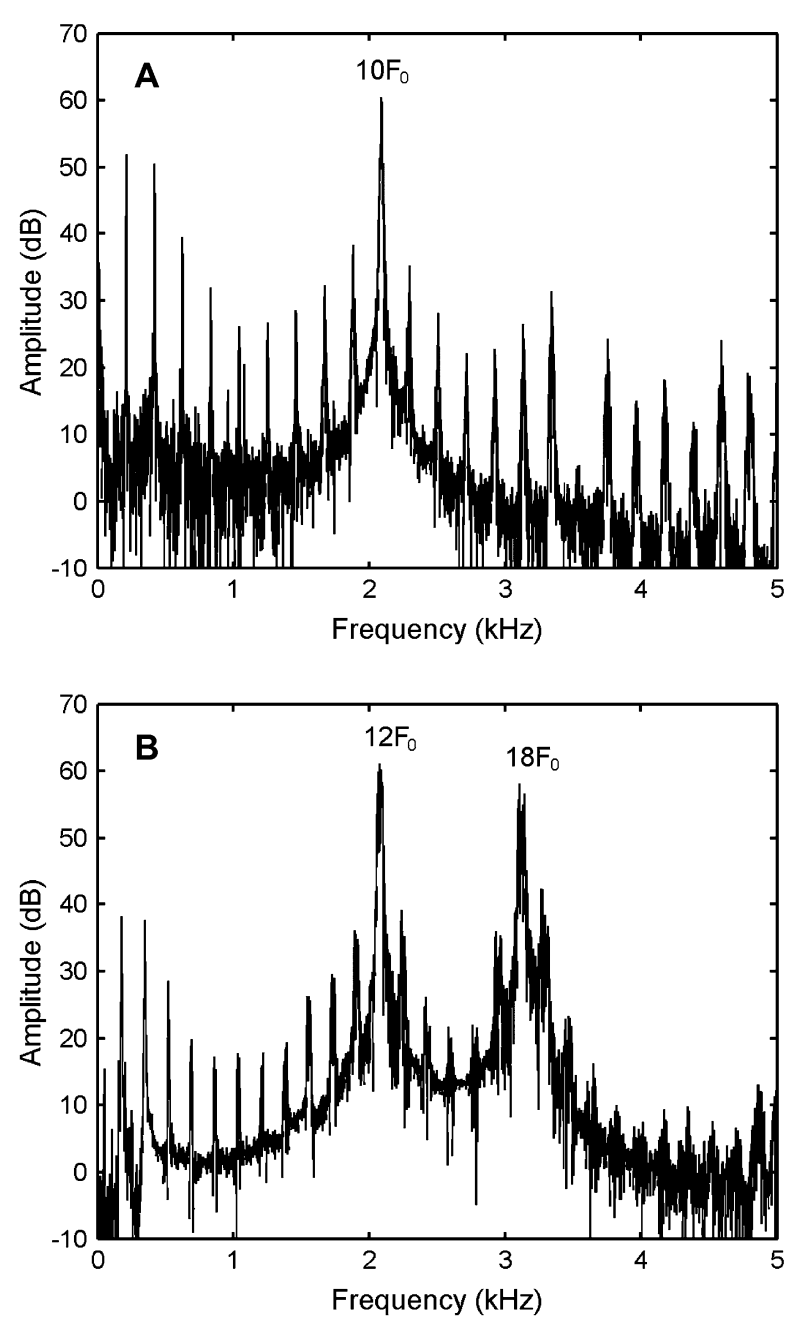

FIGURE 5. Frequency spectra of voices produced by a Taiwanese overtone singer (A) and a Tuvan overtone singer (B).

orifice was made of rubber, which is relatively stiff and its vibration amplitude was most likely negligible. In our study, the surface vibration amplitude of the supraglottal mucosa was estimated to be on the order of $0.1 \mathrm{~mm}$.

\section{High-frequency pure tone vocalization of mammals and overtone singing}

Whistle-like or "whine" sounds have been reported in anesthetized domestic dogs during electrical midbrain stimulation, ${ }^{3}$ in African wild dogs, ${ }^{5}$ and in excised larynx experiments. ${ }^{4}$ As the frequency of whines of dogs ranged from 3 to $4 \mathrm{kHz}$, it is interesting to compare the mechanisms underlying the canine whine and the human $4-\mathrm{kHz}$ vocalization. It has been postulated that female or child's flageolet voices and dog's whines are produced by vortex-induced vibrations of the vocal folds, and the periodic generation of vortices stabilized by resonances induces a periodic lifting force on the vocal folds and makes the vocal folds vibrate. $^{4,7}$ Recent experiment demonstrates that the diffuser jet without the presence of any resonator can produce $5 \mathrm{kHz}$ tones. ${ }^{15}$ The effects of suband supraglottal resonances may not be essential in the $4-\mathrm{kHz}$ vocalization. This also distinguishes the $4-\mathrm{kHz}$ vocalization from human whistling, which is due to a coupling between vortex shedding at the lips and the Helmholtz resonator of the mouth. $^{18}$

This study may also provide a look into the puzzling mechanisms underlying overtone singing. The double-source theory ${ }^{10}$ and the resonance theory ${ }^{9,19}$ have been proposed to explain the production mechanisms of this unique singing voice. Most of the techniques in overtone singing require supraglottal contraction to produce a "pressed" drone voice. 9,10 Our study on the $4-\mathrm{kHz}$ vocalization demonstrated that high-frequency tones may be produced at the narrow conduit in the larynx. It may be possible that a diffuser jet serves as the second sound source in some overtone singers' larynges. Figure 5 shows the sound spectra of overtone singing voices of two different singers. The amplified overtones are stronger than their flanking components by approximately $25 \mathrm{~dB}$. In the spectrum shown in Figure 5B, a sharp spectral peak appears at the 18th harmonic. The vorticity bursts in the larynx may play a role in the production of this overtone. Therefore, it can be hypothesized that three mechanisms may be involved in overtone singing voice production: (1) the vocal fold vibration at $F_{0}$, (2) the high-frequency vorticity bursts formed at a narrow air conduit confined by the supraglottal structures, and (3) a strong vocal tract resonance.

\section{CONCLUSION}

This study investigated the laryngeal mechanisms in human $4-\mathrm{kHz}$ vocalization. Reconstructed CT images, videostrobolaryngoscopy, and CDI were used to depict the laryngeal configuration and 
demonstrated the small-amplitude mucosal vibration on the supraglottal structures. The previously confirmed phenomenon of diffuser jet and vortexinduced vibration may be applied to explain the laryngeal mechanisms in producing the $4-\mathrm{kHz}$ vocalization.

Acknowledgments: This research was supported by Grant NSC 93-2811-B-002-079 and NSC 93-2314B-002-113, Taiwan.

\section{REFERENCES}

1. Goller F, Larsen ON. A new mechanism of sound generation in songbirds. Proc Natl Acad Sci. 1997;94:1478714791.

2. Janik VM. Whistle matching in wild bottlenose dolphins (Tursiops truncatus). Science. 2000;289:1355-1357.

3. Solomon NP, Luschei ES, Liu K. Fundamental frequency and tracheal pressure during three types of vocalizations elicited from anesthetized dogs. J Voice. 1995;9:403-412.

4. Berry DA, Herzel H, Titze IR, Story BH. Bifurcations in excised larynx experiments. J Voice. 1996;10:129-138.

5. Wilden I, Herzel H, Peters G, Tembrock G. Subharmonics, biphonation, and deterministic Chaos in mammal vocalization. Bioacoustics. 1998;9:171-196.

6. Miller DG, Schutte HK. Physical definition of the "flageolet register". J Voice. 1993;7:206-212.

7. Herzel H, Reuter R. Whistle register and biphonation in a child's voice. Folia Phoniatr Logop. 1997;49:216-224.

8. Neubauer J, Edgerton M, Herzel H. Nonlinear phenomena in contemporary vocal music. J Voice. 2004;18:1-12.
9. Adachi S, Yamada M. An acoustical study of sound production in biphonic singing, Xöömij. J Acoust Soc Am. 1999; 105:2920-2932.

10. Chernov B, Maslov V. Larynx double sound generator. Proc. XI Congress of Phonetic Sciences. Tallinn. 1987;6: 40-43.

11. Shau YW, Wang CL, Hsieh FJ, Hsiao TY. Noninvasive assessment of vocal fold mucosal wave velocity using color Doppler imaging. Ultrasound Med Biol. 2001;27:14511460.

12. Hsiao TY, Wang CL, Chen CN, Hsieh FJ, Shau YW. Elasticity of human vocal folds measured in vivo using color Doppler imaging. Ultrasound Med Biol. 2002;28:11451152.

13. Solomon NP, McCall GN, Trosset MW, Gray WC. Laryngeal configuration and constriction during two types of whispering. J Speech Hear Res. 1989;32:161-174.

14. Lell MM, Greess H, Hothorn T, Janka R, Bautz WA, Baum U. Multiplanar functional imaging of the larynx and hypopharynx with multislice spiral CT. Eur Radiol. 2004;14:2198-2205.

15. Zhang Z, Mongeau L, Frankel SH, Thomson S, Park JB. Sound generation by steady flow through glottis-shaped orifices. J Acoust Soc Am. 2004;116:1720-1728.

16. Tsunoda K, Ohta Y, Soda Y, Niimi S, Hirose H. Laryngeal adjustment in whispering magnetic resonance imaging study. Ann Otol Rhinol Laryngol. 1997;106:41-43.

17. Hofmans GCJ, Groot G, Ranucci M, Graziani G, Hirschberg A. Unsteady flow through in-vitro models of the glottis. J Acoust Soc Am. 2003;113:1658-1675.

18. Wilson TA, Beavers GS, DeCoster MA, Holger DK, Regenfuss MD. Experiments on the fluid mechanics of whistling. J Acoust Soc Am. 1971;50:366-372.

19. Kob M. Analysis and modeling of overtone singing in the sygyt style. Appl Acoust. 2004;65:1249-1259. 